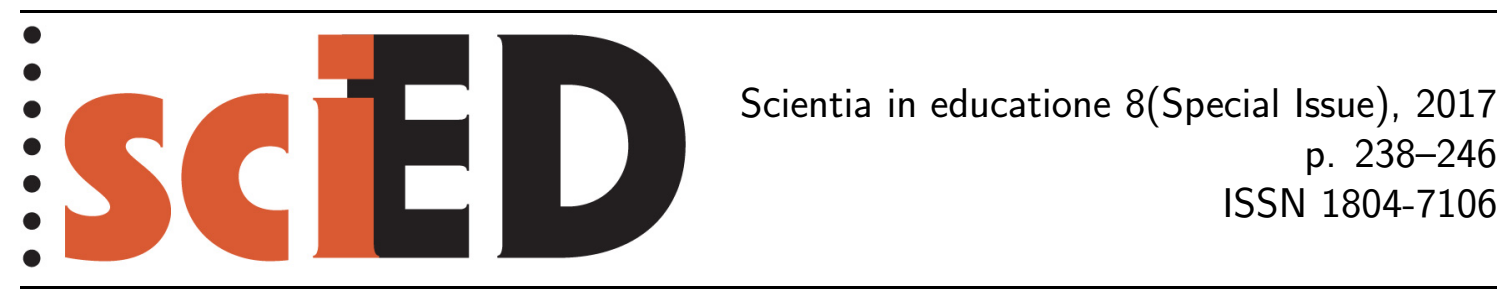

\title{
Chaos at High School
}

\section{Tamás Meszéna}

\begin{abstract}
We are faced with chaotic processes in many segments of our life: meteorology, environmental pollution, financial and economic processes, sociology, mechanics, electronics, biology, chemistry. The spreading of high-performance computers and the development of simulation methods made the examination of these processes easily available. Regular, periodic motions (pendulum, harmonic oscillatory motion, bouncing ball), as taught at secondary level, become chaotic even due minor changes. If it is true that the most considerable achievements of twentieth century physics were the theory of relativity, quantum mechanics and chaos theory, then it is presumably time to think about, examine and test how and to what extent chaos can be presented to the students. Here I would like to introduce a 12 lesson long facultative curriculum framework on chaos designed for students aged seventeen. The investigation of chaos phenomenon in this work is based on a freeware, "Dynamics Solver". This software, with some assistance from the teacher, is suitable for classroom use at secondary level.
\end{abstract}

Key words: chaotic process, numerical simulation, nonlinear oscillators, Dynamics Solver. 


\section{INTRODUCTION}

It was a common opinion at the end of the 19th century that, in physics, what could be found out, it had been done. For this reason, the young Max Planck was advised by his teacher to choose some other profession, not physics (Planck, 1958). However, it didn't take more than a few years, and a convincing reply was given to this thoughless opinion. Since then no physicist would think that physics will ever be fully known. Nevertheless, it seems, the same mistake has been made again and again. At the end of the 20th century, perhaps, not many people expected any discoveries in classical physics. But chaos theory is just such a thing.

\section{TEACHING CHAOTIC PHENOMENA AT HIGH SCHOOL}

\section{WHY TO TEACH CHAOS AT HIGH SCHOOL?}

Chaos, in mechanical motion, for example, is not just a scientific peculiarity. In contrast, chaotic motion is found nearly everywhere, if our world is investigated in fine detail.

"From 20th century science three concepts will be remembered only: theory of relativity, quantum mechanics and chaos theory." wrote James Gleick in his book, Chaos (Gleick, 1988). It might be an exaggeration to some extent, since while the first two theories brought new equations of motion, basically, the theory of chaos revealed new depths of an equation of motion known for a long time. The fundamental observations of the first two theories (born 90-110 years ago) have been incorporated in secondary education curriculum. The real development of chaos theory started some 30 years ago. The investigation of chaotic phenomena has brought such a fundamental change in the interpretation of nature, that is undoubtedly reasonable and, luckily, possible to deal with in secondary education. However, very few secondary school text book includes these subjects. In Hungary, actually, there is none at all. For this reason, a syllabus suitable for facultative classes is suggested in this work.

Regular motions tought in secondary school, strictly speaking, do not exist in nature. They can be treated as exceptions, perhaps. Chaotic motion is widely spread. It takes an eager pupil to follow text book concepts enthusiasticly. Others loose interest towards physics partly due to the many simplifying assumptions made (needed be able to describe motion mathematically). As a result, pupils do not feel that their real-life observations would be delt with in physics classes. It is a real joy to both teacher and pupils when such a subject is lectured that is possible to observe in nature approximately in the same way as in the theory.

Chaos is interesting, beautiful and it has the sense of mistery. Features that come very useful in education. Some of the first occasions when the subject is mentioned for an average pupil probably include the chaos researcher, a main character of Steven Spielberg's film, Jurassic Park, or the fractals of sprawling plants mimicing human soul in Paul Young's novel, The Shack.

In Figure 1 the path of a magnetic pendulum above a plane containing three attractive magnets can be seen. Figure 2 shows the complex geometric structure characteristic of chaos in the mixing process of dyes. 


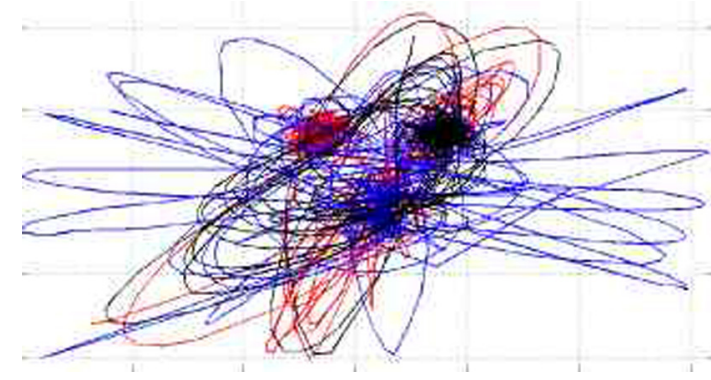

Figure 1: Path of a magnetic pendulum

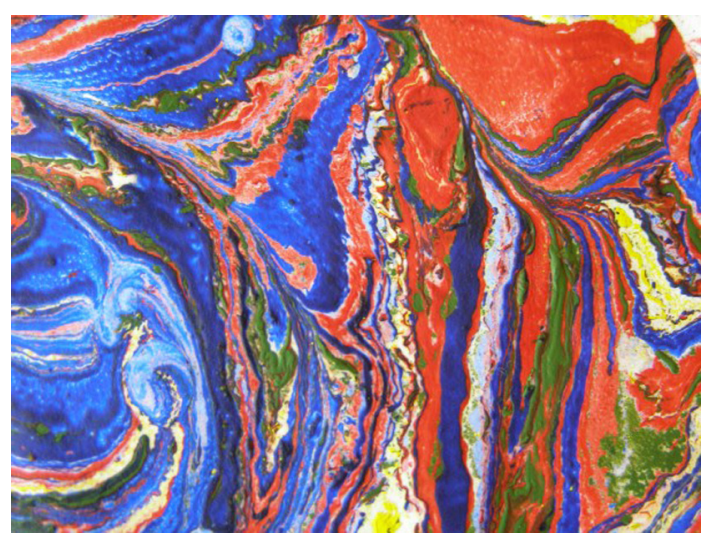

Figure 2: Chaotic mixing of dyes

\section{WHAT TO TEACH FROM CHAOS THEORY?}

Let us consider a few simple examples where regular motion studied at school becomes chaotic with little modification. Pendulum motion shows this behaviour. The motion of a simple pendulum is regular. However, if the point of suspension is moved periodically (driven pendulum), or two pendulums are coupled (double pendulum), or magnets are placed next to the pendulum (magnetic pendulum), see Figure 3, the motion becomes typically chaotic.
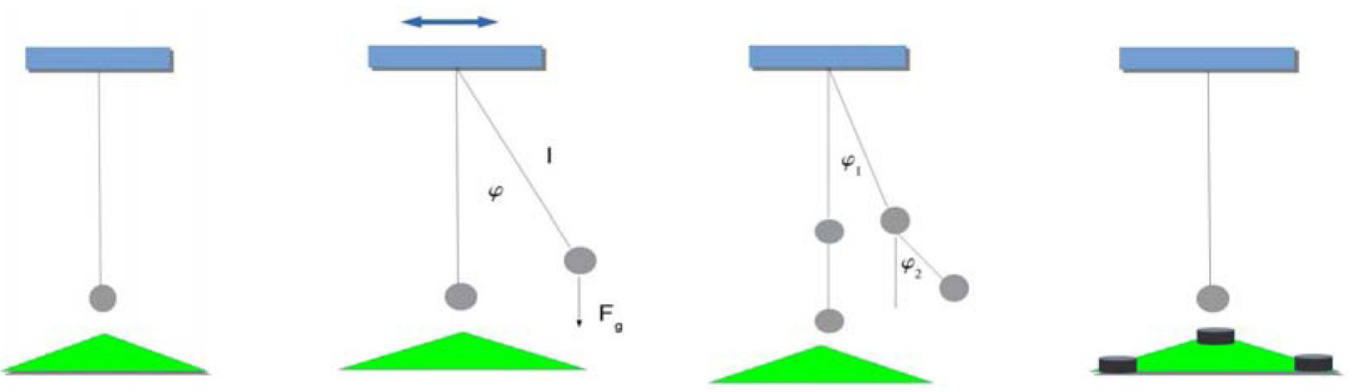

Figure 3: Mathematical pendulum, driven pendulum, double pendulum, magnetic pendulum. The latter three systems are chaotic

Another example of a simple regular motion is a bouncing ball on the table. A bouncing ball on an oscillating plate, on a double edge or on stairs (Figure 4) may turn to be chaotic.
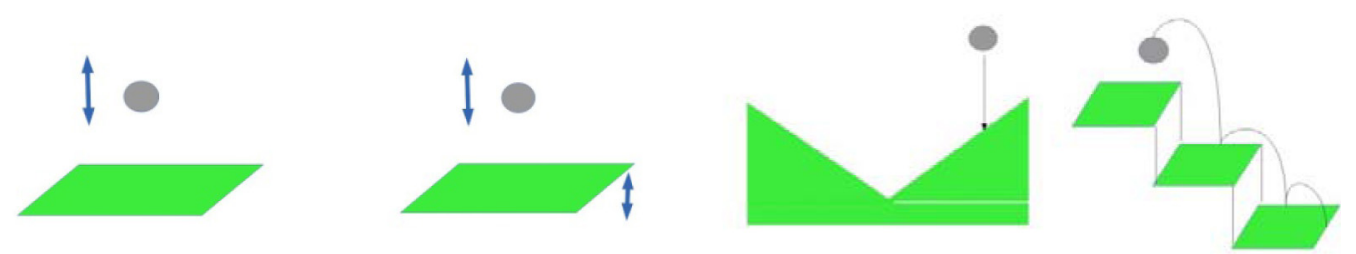

Figure 4: Bouncing ball, bouncing ball on an oscillating plate, on a double edge, on a stairway. The latter three systems are chaotic

Let us investigate the motion of a driven oscillator in detail. If a driven oscillator is based on a spring that obeys Hooke's law, the resulting motion will be a regular motion that is easy to describe. With a nonlinear spring (Figure 5), however (and real springs are never perfectly linear), motion may become much more complicated: it may show chaotic behaviour. 


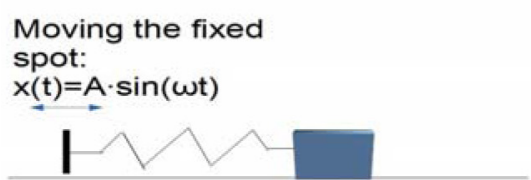

Linear spring

Regular motion
Moving the fixed spot:

$x(t)=A \cdot \sin (\omega t)$

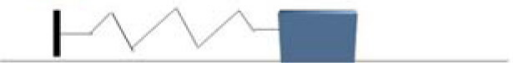

Nonlinear spring

Chaotic motion

Figure 5: In spite of the identical sinusoidal driving, the motion of a body fixed to a linear spring is always regular, whereas that with a nonlinear (i.e. realistic) spring is typically chaotic

The description of chaotic motion can be well explained by the concepts used for the example of nonlinear oscillators. Regular motions are normally described in terms of position versus time, velocity versus time (and acceleration versus time) functions. In case of chaotic motions, these functions are so complicated and irregular that by graphing these functions it is hard to recognize the surprising order that is inherent in these motions. Before chaos theory appeared, such motions were simply considered irregular. A more appropriate representation is needed that reflects the properties of such motions better, and makes it possible to reveal the order underlying chaos.

No information or systematic behaviour can be deduced from the investigation of the usual functions since the periodicity of the original oscillator is entirely lost (Figure 6).
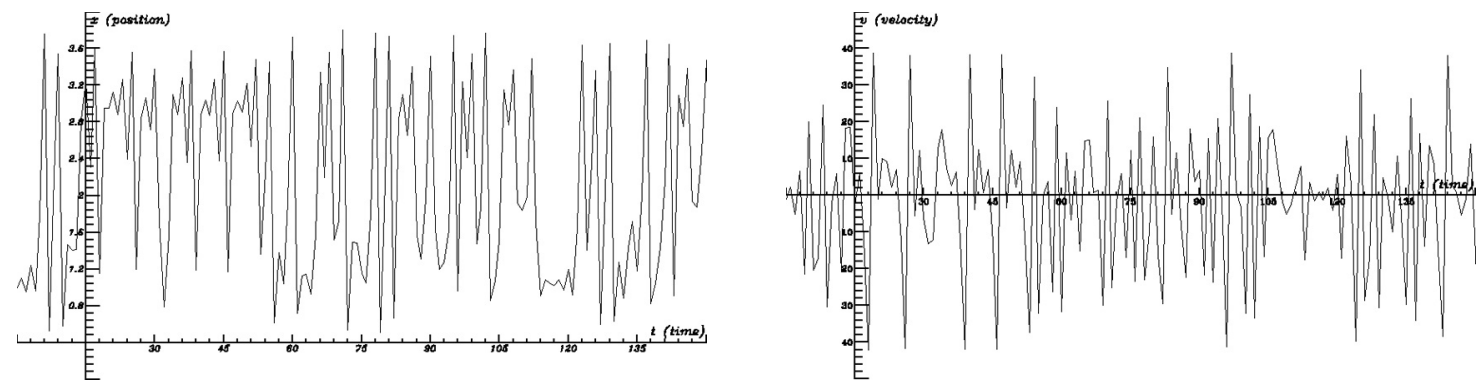

Figure 6: Position-time and velocity-time representation of a driven nonlinear oscillator

Information needs to be condensed and represented in another way. To represent chaotic motion, a velocity versus position graph (called phase space) is used, since it provides a better overview of such motions. The complicated geometrical structure, characteristic of the motion, is revealed by taking samples at regular intervals (at that of the driving period), and by plotting only these points on the plane of the phase space (Figure 7). This procedure is called a stroboscopic map.

Note that in this representation the image of a periodic motion is a single point, since the location and the velocity would be always the same, period by period. It is obvious from Figure 7 that, although chaotic motion never repeats itself, it cannot be considered completely irregular, it is not like white noise. It has a clean-cut, profound order, just a much more complex one than that of the periodic motions.

Chaos can be described as the "complex temporal behaviour of simple systems" 


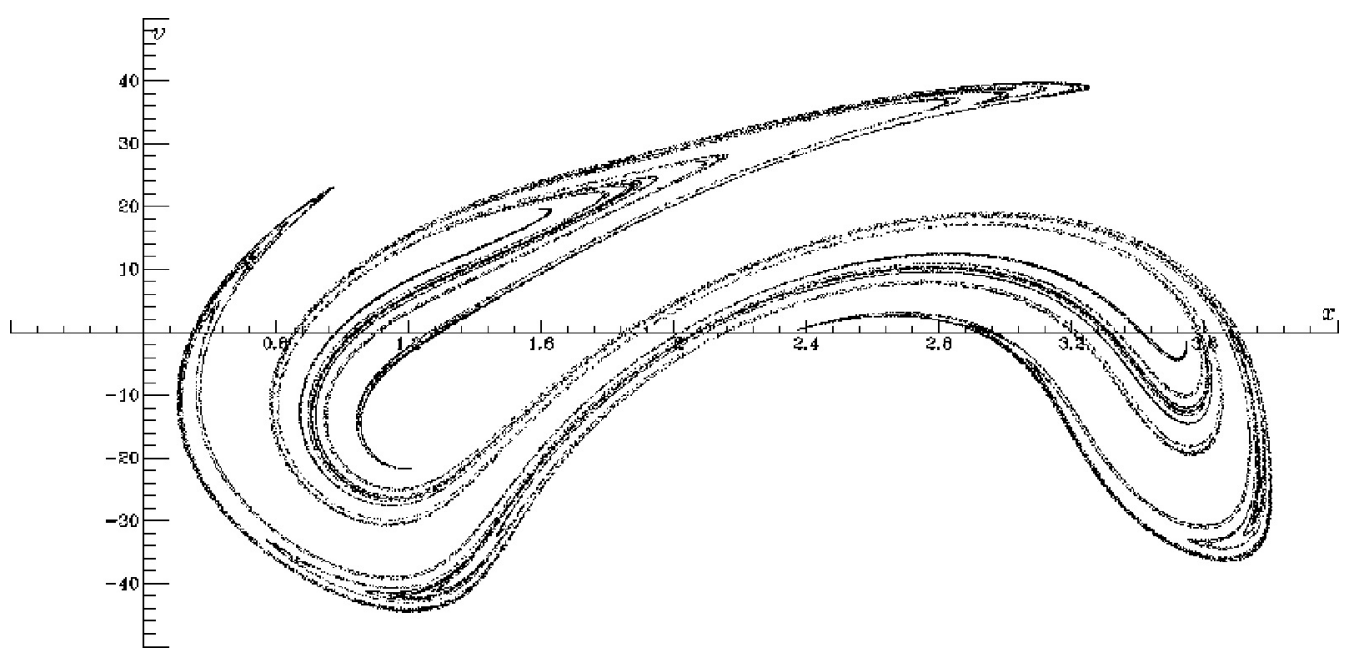

Figure 7: The stroboscopic mapping on the phase space of a driven nonlinear oscillator. This presentation exhibits the unusual pattern underlying chaotic motion

(Ott, 1993; Tél \& Gruiz, 2006). It is highly instructive to realize the fact that even simple mechanical systems, known for a long time, can be chaotic.

In the following section the focus is on a method we propose to produce, in cooperation with the pupils, Figure 7, and make them understand the equation of motion used for this phenomenon.

The equation of motion for the position $x$ of the driven nonlinear oscillator is:

$$
\ddot{x}=-\omega_{0}^{2} x+\varepsilon x^{3}-\alpha \dot{x}+\mathrm{A}_{0} \cos \omega t .
$$

Here $\ddot{x}$ is the second time-derivative of the position, $\omega_{0}$ is the frequency of the oscillator, $\varepsilon$ is the parameter of the nonlinear spring, $x^{\prime}$ is the velocity, $\alpha$ is the parameter of the drag coefficient, and $\mathrm{A}_{0}$ and $\omega$ are the amplitude and frequency of the driving, respectively.

Secondary school pupils are not aware of differential equations, most probably they don't even know what differential calculus is. However, the meaning of the above equation can be unfolded by using the concept of velocity $(v)$ and acceleration (a) instead of time derivatives. With properly chosen units the above equation can be written in a simpler form:

$$
a=-A^{2} x-A^{2} x^{3}-B v+C \cos (2 \pi t)
$$

Measuring time in units of driving period $2 \pi / \omega$ and distance in suitable units, quantity a $(v)$ represents the dimensionless acceleration (velocity). Parameters $A$, $B$ and $C$ are numbers characterizing the spring's strength, the drag and the driving amplitude, respectively. In this form the physical meaning of the four terms on the right hand side are: linear and nonlinear spring force, drag, and driving. This way a sufficient interpretation is given for the equation, even if not all details are fully explained.

For the description of various dynamics two fundamentally different methods are used:

1. Forces acting on the object are known, and the functions describing the path can be explicitly given. Everything is known, basically. Examples are motion with constant acceleration, and harmonic oscillation:

$$
\begin{aligned}
& a=\frac{F}{m} \text { const } \Rightarrow s(t)=\frac{a}{2} t^{2}+v_{0} t \\
& a=-\omega^{2} y \Rightarrow y(t)=A \sin \left(\omega t+\phi_{0}\right)
\end{aligned}
$$


The trajectory cannot be explicitly given due to nonlinear forces, it can be numerically calculated only, step by step. This is the case for chaotic motion.

A numerical solution should be performed, for example, by means of the Dynamics Solver program.

\section{The Dynamics Solver PROGRAM}

Dynamics Solver is a freeware, developed for the numerical integration of sets of differential equations by Juan M. Aguirregabiria in Spain. The software can be freely downloaded from several websites (see e.g. Dynamics Solver).

Input data for the calculations are the number of equations, the number and notation of variables, functional relationships, parameters of theequation, initial conditions, and the parameters of the visualization.

Second order equations are solved as a set of first order equations. The format of equations is the one used in secondary schools:

$$
\begin{aligned}
& \frac{\mathrm{d} x}{\mathrm{~d} t}=v \\
& \frac{\mathrm{d} v}{\mathrm{~d} t}=-A^{2} x-A^{2} x^{3}-B v+C \cos (2 \pi t)
\end{aligned}
$$

The Dynamics Solver program starts with the screen shown in Figure 8: here the various parameters and initial values can be specified and, at the same time, the results of calculations are presented. The chaotic attractor of Figure 7 was obtained with $A=6, B=0.6, C=1800$. The initial values were taken as $t_{0}=0$, $x_{0}=1, v_{0}=-1$ and the simulation was run over 1000000 dimensionless time units. The chaotic attractor is reached after about a single time unit, the pattern seen in Figure 7 is therefore independent of the initial conditions.

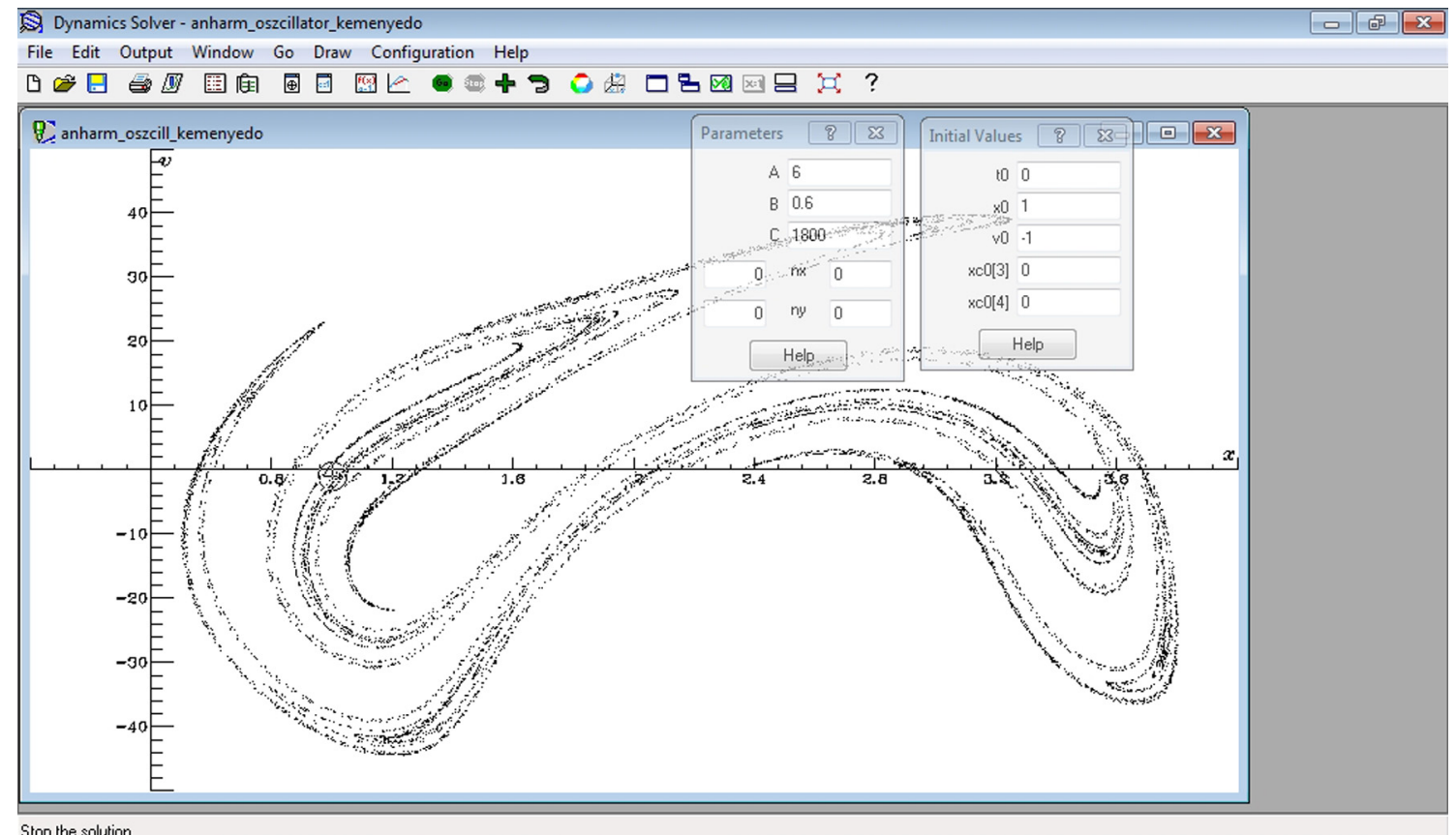

Figure 8: The screen of the Dynamics Solver program. The boxes in the right upper corner contain the parameters and the initial values. The data appearing with zero values in the boxes are irrelevant for our purposes 
The program is user friendly, easy to understand. The only problem to overcome is the huge number of choices.

The graphs shown in Figure 7 and 8 have been produced by specifying the presented results as snapshots taken in every driving period on the $x-v$ plane. The resulting complex shape is called the chaotic attractor. The filamentary structure of the attractor is infinitely complex, it is a fractal.

Once the necessary equations have been entered and settings have been made, it is very easy to test the effect of changing parameters and initial conditions. This can serve as the pupils' own research activity, even with the possibility of some significant achievements.

Various motions can be studied with the method described. First, motions studied in the standard curriculum should be simulated. Results already known can be checked and, at the same time, sufficient skills can be gained in using the software. Once the software is familiar, various chaotic motions can be studied.

\section{SyllabUS OF A CHAOS-TEACHING PROGRAM}

Facultative program for 17 year old students with a sum of 12 teaching hours. The topics of the classes are the following:

Class 1-2. Introduction, demonstrating a few chaotic phenomena: chaotic pendulums, bouncing balls, driven nonlinear oscillator. Equations of motions, solving simple equations of motion. Homework: home-made chaotic tools.

Class 3-4. Examination of chaos with computer: generating chaotic attractors (of e.g. the driven pendulum), discussing the necessary concepts: chaos, phase space, stroboscopic mapping, attractors. Solving differential equations numerically by means of Dynamics Solver.

Class 5-6. Fractal properties of chaotic attractors. Mathematical fractals, interpretation of fractal dimension, examples. Physical fractals, examples from biology and geography.

Class 7-8. Examination of further chaotic phenomena, computer simulations.

Class 9-10. Computer simulations. Chaotic phenomena in other disciplines: biology, chemistry, meteorology, geography, astronomy, sociology and economy.

Class 11-12. Summary, "final assessment". Summing up experiences, discussion.

\section{AtTempts TO TEACH CHAOS IN HUNGARY AND IN OTHER COUNTRIES}

In 2003 Ildikó Szatmári-Bajkó developed a similar chaos teaching program, based on the use of a chaotic motion simulation program, developed at the Department of Theoretical Physics of Eötvös University (Szatmári-Bajkó, 2010, 2006). In 2008 and 2010 József Jaloveczki published articles on numerically solving equations of motion with students at high schools (Eichhardt \& Jaloveczki, 2008, 2009).

In Italy, I am aware of two books which mention chaotic phenomena, such as molecular and deterministic chaos (Caforio \& Ferilli, 1993; Violino \& Robutti, 1995). The basic characteristic features of a chaotic system are mentioned in a textbook of physics in Austria (Jaros, Nussbaumer \& Kunze, 1999). Some elements of chaos theory are relatively detailed in a textbook in Romania (Tellmann, Darvay \& Kovács, 2006). 


\section{CONCLUSIONS}

It's worth teaching chaos theory at high school level since it gives an insight for the students into a recently discovered feature of physics, this may raise further the interest towards science. The widespread popularity of informatics also helps us to direct students towards an important field, to give opportunity of creative work, and to provide artistic facets of scientific activities.

The Dynamics Solver freeware is an appropriate program for work with students. Its use is simple and easily overcomes any lack of knowledge in math acquired in secondary school. It gives opportunity to design independent experiments and may lead to results, as well as, to aesthetic joy.

It's a striking experience seeing how the chaotic attractor of a driven nonlinear oscillator or pendulum emerges from thousands of points on the screen. The latter is shown in Figure 9.

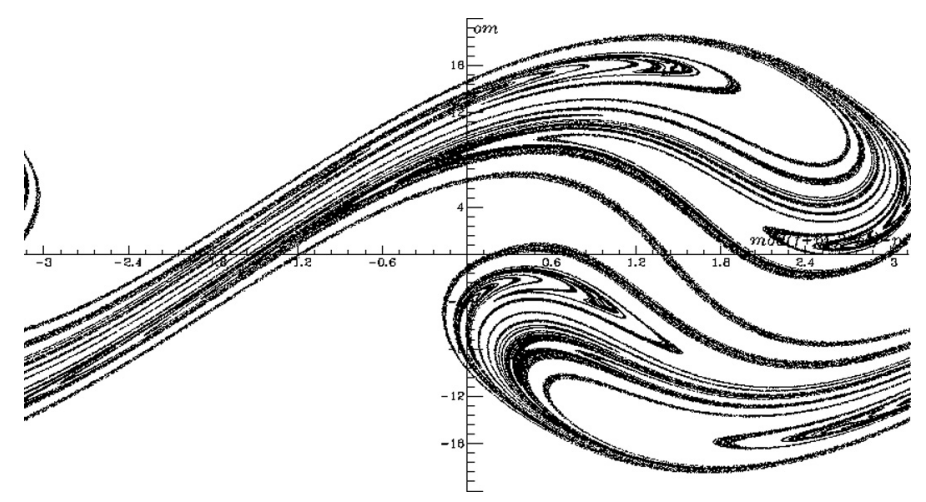

Figure 9: The chaotic attractor of a driven pendulum in the stroboscopic map generated by Dynamics Solver

The equation of motion of the driven pendulum is:

$$
\ddot{\phi}=-\frac{g}{l} \sin (\phi)-\alpha \dot{\phi}+\frac{A \omega^{2}}{l} \cos (\omega t) \cos (\phi)
$$

Measuring time in units of the driving period and distance in the unit of the pendulum's length $l$, three parameters remain. The dimensionless equation of motion is:

$$
\ddot{\phi}=-a \sin (\phi)-b \dot{\phi}+c \cos (2 \pi t) \cos (\phi)
$$

Parameters $a, b, c$ are numbers characterizing the frequency, the drag and the driving amplitude, respectively. The simulation is run with $a=4 \pi^{2} / 9, b=0.2 \pi$, $c=8 \pi^{2}$, and with the initial values $\phi_{0}=1$ and $\omega_{0}=0$ for a time of 1000000 dimensionless units.

\section{ACKNOWLEDGEMENT}

Special thanks are due to my PhD supervisors Márton Gruiz and Tamás Tél for their useful pieces of advice. 


\section{REFERENCES}

Caforio, A. \& Ferilli, A. (1993). Physica 2. Firenze: Le Monier.

Dynamics Solver. Available at http://tp.lc.ehu.es/jma/ds/ds.html

Eichhardt, I. \& Jaloveczki, J. (2008). Fizikázzunk egyszerûen, számítógéppel (Let's study physics the simplest way: using only a computer). Fizikai Szemle, 2008(9), 311-315.

Eichhardt, I. \& Jaloveczki, J. (2009). Numerikus módszerek a diákköri munkában (Numerical methods in students' circle work). Fizikai Szemle, 2009(10), 348-351.

Gleick, J. (1988). Chaos. Making a new science. Penguin Books.

Jaros, A., Nussbaumer, A. \& Kunze, H. (1999). Basiswissen Physik-compact. Wien: Öbv\&hpt.

Ott, E. (1993). Chaos in dynamical systems. Cambridge University Press.

Planck, M. (1958). Physikalische Abhandlungen und Vortrage. Braunschweig: Friedrich Vieweg und Sohn.

Szatmári-Bajkó, I. (2010). Káosz, rend, látvány. Iskolakultúra, 2010(1), 116-131.

Szatmári-Bajkó, I. (2006). "Káoszt"? - azt! (Some chaos? Yes, let us have it!), Fizikai Szemle, 2006(11), 376-380.

Tél, T. \& Gruiz, M. (2006). Chaotic dynamics. Cambridge University Press.

Tellmann, J., Darvay, B. \& Kovács, Z. (2006). Fizika XI. Ábel Kiadó.

Violino, P. \& Robutti, O. (1995). La fisica e i suio modelli, Volume 3. Bologna:

Zanichelli.

TAMÁS MESZÉNA

Nagy Lajos High School of the Cistercian Order, 7621 Pécs Széchenyi tér 11, Hungary

Physics Education PhD Program Eötvös University, Budapest, Hungary 